\title{
The critical exponent for fast diffusion equation with nonlocal source
}

\author{
Chunxiao Yang ${ }^{1 *} \mathbb{D}$, Linghua Kong ${ }^{2}$, Yingxue $\mathrm{Wu}^{1}$ and Qing Tian ${ }^{1}$
}

"Correspondence:

chunxiao_yang@aliyun.com

'School of Science, Xi'an University of Architecture and Technology,

Xi'an, P.R. China

Full list of author information is available at the end of the article

\begin{abstract}
This paper considers the Cauchy problem for fast diffusion equation with nonlocal source $u_{t}=\Delta u^{m}+\left(\int_{\mathbb{R}^{n}} u^{q}(x, t) d x\right)^{\frac{p-1}{q}} u^{r+1}$, which was raised in [Galaktionov et al. in Nonlinear Anal. 34:1005-1027, 1998]. We give the critical Fujita exponent $p_{c}=m+\frac{2 q-n(1-m)-n q r}{n(q-1)}$, namely, any solution of the problem blows up in finite time whenever $1<p \leq p_{c}$, and there are both global and non-global solutions if $p>p_{c}$.
\end{abstract}

MSC: 35B33; $35 \mathrm{~K} 65$

Keywords: Critical exponents; Fast diffusion; Nonlocal; Global solutions; Blow-up

\section{Introduction}

In this paper, we study the following Cauchy problem of fast diffusion parabolic equation with a nonlinear nonlocal source:

$$
\begin{cases}u_{t}=\Delta u^{m}+\left(\int_{\mathbb{R}^{n}} u^{q}(x, t) d x\right)^{\frac{p-1}{q}} u^{r+1}, & (x, t) \in \mathbb{R}^{n} \times(0, T), \\ u(x, 0)=u_{0}(x), & x \in \mathbb{R}^{n},\end{cases}
$$

where the spatial dimension $n \geq 1$, the coefficients $m, p, q, r$ satisfy $\max \left\{0,1-\frac{2}{n}+r\right\}<$ $m<1, p>1, q \geq 1,0 \leq r<\frac{2}{n}$, and the initial data $u_{0}(x)$ is a nontrivial nonnegative continuous function.

The quasilinear parabolic equations involving a nonlocal term originate in the phenomena of diffusion about concentration of some Newtonian fluids or the density of some biological species and heat transfer in a special medium with nonlocal source (see $[2,3]$ and the references therein). In the past three decades, various nonlocal mathematical models were established to describe many physical phenomena (see [1, 4-9] and references therein). At the same time, many important results have appeared on the blow-up problem for a nonlinear parabolic equation with nonlocal source (see [2, 6, 8-11] and references therein), and for nonlocal nonlinear diffusion equations [12,13]. However, most of efforts have been devoted in bounded domains, there were few researches for the Cauchy problems (see $[1,14,15])$.

It is well known that the classical Cauchy problem

$$
u_{t}=\Delta u+u^{p} \quad \text { in } \mathbb{R}^{n} \times(0, T)
$$

(c) The Author(s) 2019. This article is distributed under the terms of the Creative Commons Attribution 4.0 International License (http://creativecommons.org/licenses/by/4.0/), which permits unrestricted use, distribution, and reproduction in any medium, provided you give appropriate credit to the original author(s) and the source, provide a link to the Creative Commons license, and indicate if changes were made. 
possesses the critical exponent $1+\frac{2}{n}$ [16-19], that is to say, any nontrivial solution blows up in finite time if $1<p \leq 1+\frac{2}{n}$, whereas global and non-global solutions coexist if $p>$ $1+\frac{2}{n}$, depending on the size of initial data. From then on, the Fujita phenomenon has been observed for many nonlinear PDEs (see surveys [20,21] and references therein).

The study for the Cauchy problem of nonlocal nonlinear parabolic equation was proposed by Galaktionov et al. [1], in which it was proved that the Cauchy problem (1.1) with $m=1$ has a critical Fujita exponent, and Wang et al. [15] obtained similar results by other methods. Recently, Zhou [14] considered the global and non-global existence of solutions for (1.1) with $m>1$.

The present paper investigates a fast diffusion parabolic equation $(1.1)\left(\max \left\{0,1-\frac{2}{n}\right\}<\right.$ $m<1)$ with a nonlocal source, and establishes the critical Fujita exponent $p_{c}=m+$ $\frac{2 q-n(1-m)-n q r}{n(q-1)}$. Comparing with the known result for the parallel problem with a local source

$$
u_{t}=\Delta u^{m}+u^{p} \quad \text { in } \mathbb{R}^{n} \times(0, T)
$$

the critical Fujita exponent was obtained in $[22,23]$ and shown to be $p_{c}=1+\frac{2 m}{n}$.

In the rest of the paper, we always let $u$ be a solution to (1.1), and $p_{c}=m+\frac{2 q-n(1-m)-n q r}{n(q-1)}$. The main results are stated in the following theorems.

Theorem 1.1 For $1<p \leq p_{c}$, there are no global nontrivial solutions to (1.1).

Theorem 1.2 For $p>p_{c}$, there are both global and non-global solutions to (1.1).

This paper is organized as follows. Section 2 concerns the non-global solution to prove Theorem 1.1. Section 3 deals with the global existence to prove Theorem 1.2. And Sect. 4 shows in what ways the parameter $q$ of the nonlocal source affects the behavior of solutions in the fast diffusion problem (1.1).

\section{Non-global solutions}

This section mainly applies the test function method (refer to $[15,22])$ to prove that any solution of (1.1) must blow up in finite time for $1<p \leq p_{c}$. Introducing the test function

$$
\varphi_{k}(x)=\left(\frac{k}{\pi}\right)^{\frac{n}{2}} \mathrm{e}^{-k|x|^{2}}
$$

for some $k>0$, we can simply verify that

$$
\int_{\mathbb{R}^{n}} \varphi_{k}(x) d x=1, \quad\left\|\varphi_{k}(x)\right\|_{L^{\infty}}=\left(\frac{k}{\pi}\right)^{\frac{n}{2}}, \quad \Delta \varphi_{k}(x) \geq-2 k n \varphi_{k}(x) .
$$

Define

$$
F(t)=\int_{\mathbb{R}^{n}} u(x, t) \varphi_{k}(x) d x .
$$

It is sufficient to show that $F(t)$ blows up in finite time as $1<p \leq p_{c}$ to deal with Theorem 1.1. 
Proof of Theorem 1.1 Firstly, we consider the case of $1<p<p_{c}$. Multiplying equation (1.1) by $\varphi_{k}(x)$ and integrating by parts in $\mathbb{R}^{n}$, we get

$$
\begin{aligned}
F^{\prime}(t) & =\int_{\mathbb{R}^{n}} u_{t} \varphi_{k} d x \\
& =\int_{\mathbb{R}^{n}} \Delta u^{m} \varphi_{k} d x+\left(\int_{\mathbb{R}^{n}} u^{q} d x\right)^{\frac{p-1}{q}} \int_{\mathbb{R}^{n}} u^{r+1} \varphi_{k} d x \\
& \geq-2 k n \int_{\mathbb{R}^{n}} u^{m} \varphi_{k} d x+\left\|\varphi_{k}\right\|_{L^{\infty}}^{-\frac{p-1}{q}}\left(\int_{\mathbb{R}^{n}} u^{q} \varphi_{k} d x\right)^{\frac{p-1}{q}} \int_{\mathbb{R}^{n}} u^{r+1} \varphi_{k} d x .
\end{aligned}
$$

Using Jensen's inequality for $m<1, q>1$, and $r>0$,

$$
\begin{aligned}
F^{\prime}(t) & \geq-2 k n F^{m}(t)+\left(\frac{k}{\pi}\right)^{-\frac{n(p-1)}{2 q}} F^{p+r}(t) \\
& =F^{p+r}(t)\left(\left(\frac{k}{\pi}\right)^{-\frac{n(p-1)}{2 q}}-2 k n F^{-(p+r-m)}(t)\right) .
\end{aligned}
$$

Assuming

$$
F(t)>\left(\pi^{\frac{n(p-1)}{2 q}}(4 n)^{-1}\right)^{-\frac{1}{p+r-m}} k^{\frac{2 q+n(p-1)}{2 q(p+r-m)}}
$$

we obtain

$$
\left(\frac{k}{\pi}\right)^{-\frac{n(p-1)}{2 q}}>4 k n F^{-(p+r-m)}(t)
$$

and

$$
F^{\prime}(t) \geq \frac{1}{2}\left(\frac{k}{\pi}\right)^{-\frac{n(p-1)}{2 q}} F^{p+r}(t)
$$

This implies

$$
F(t) \geq\left(F^{-(p+r-1)}(0)-\frac{p+r-1}{2}\left(\frac{k}{\pi}\right)^{-\frac{n(p-1)}{2 q}} t\right)^{-\frac{1}{p+r-1}}
$$

Obviously, $F(t)$ blows up for any nonnegative initial data as $t \rightarrow T=\frac{2 F^{-(p+r-1)}(0)}{p+r-1}\left(\frac{k}{\pi}\right)^{\frac{n(p-1)}{2 q}}$. In the following, we show that

$$
F(0)>\left(\pi^{\frac{n(p-1)}{2 q}}(4 n)^{-1}\right)^{-\frac{1}{p+r-m}} k^{\frac{2 q+n(p-1)}{2 q(p+r-m)}}
$$

is a sufficient condition to prove condition (2.2). If not, there exists some $\tau$, such that

$$
F(\tau)=\left(\pi^{\frac{n(p-1)}{2 q}}(4 n)^{-1}\right)^{-\frac{1}{p+r-m}} k^{\frac{2 q+n(p-1)}{2 q(p+r-m)}}
$$


and

$$
F(t)>\left(\pi^{\frac{n(p-1)}{2 q}}(4 n)^{-1}\right)^{-\frac{1}{p+r-m}} k^{\frac{2 q+n(p-1)}{2 q(p+r-m)}}, \quad t \in[0, \tau) .
$$

This implies $F^{\prime}\left(\tau_{0}\right)<0$ for some $\tau_{0} \in(0, \tau)$, which contradicts $F^{\prime}(t) \geq 0, t \in(0, \tau)$. Thereby, to prove that a solution of (1.1) blows up in finite time, we only show (2.4) is true for any nonnegative nontrivial initial data $u_{0}(x)$. Since $\frac{n}{2}<\frac{2 q+n(p-1)}{2 q(p+r-m)}$ which was derived by $1<p<$ $p_{c}$, there exists a $k>0$ small enough, such that

$$
F(0)=\left(\frac{k}{\pi}\right)^{\frac{n}{2}} \int_{\mathbb{R}^{n}} \mathrm{e}^{-k|x|^{2}} u_{0}(x) d x>\left(\pi^{\frac{n(p-1)}{2 q}}(4 n)^{-1}\right)^{-\frac{1}{p+r-m}} k^{\frac{2 q+n(p-1)}{2 q(p+r-m)}} .
$$

Next, we consider the case of $p=p_{c}$. Supposing a solution of (1.1) is global for any $t \geq 0$, it holds that

$$
F(t)=\left(\frac{k}{\pi}\right)^{\frac{n}{2}} \int_{\mathbb{R}^{n}} \mathrm{e}^{-k|x|^{2}} u(x, t) d x \leq\left(\pi^{\frac{n(p-1)}{2 q}}(4 n)^{-1}\right)^{-\frac{1}{p+r-m}} k^{\frac{2 q+n(p-1)}{2 q(p+r-m)}} .
$$

That is, if (2.5) is not true, namely $F\left(t_{1}\right)>\left(\pi^{\frac{n(p-1)}{2 q}}(4 n)^{-1}\right)^{-\frac{1}{p+r-m}} k^{\frac{2 q+n(p-1)}{2 q(p+r-m)}}$ for some $t_{1}>0$, then the solution $u(x, t)$ must blow up in finite time by the above proof. The condition $p=p_{c}$ means $\frac{n}{2}=\frac{2 q+n(p-1)}{2 q(p+r-m)}$, and (2.5) can be rewritten as

$$
\int_{\mathbb{R}^{n}} \mathrm{e}^{-k|x|^{2}} u(x, t) d x \leq \pi^{\frac{n}{2}}\left(\pi^{\frac{n(p-1)}{2 q}}(4 n)^{-1}\right)^{-\frac{1}{p+r-m}} \quad \text { for } t>0
$$

Without loss of generality, assuming $u_{0}(x)$ has compact support in $\mathbb{R}^{n}$, we get that $u(x, t) \in$ $L\left(\mathbb{R}^{n}\right)$ for any fixed $t>0$ (see [24]). By Lebesgue Dominated Convergence Theorem, as $k \rightarrow 0$ in (2.6),

$$
\int_{\mathbb{R}^{n}} u(x, t) d x \leq \pi^{\frac{n}{2}}\left(\pi^{\frac{n(p-1)}{2 q}}(4 n)^{-1}\right)^{-\frac{1}{p+r-m}}
$$

Integrating equation $(1.1)$ on $\mathbb{R}^{n} \times[0, t]$, we have

$$
\int_{\mathbb{R}^{n}} u(x, t) d x-\int_{\mathbb{R}^{n}} u_{0}(x) d x=\int_{0}^{t}\left(\int_{\mathbb{R}^{n}} u^{q} d x\right)^{\frac{p-1}{q}} \int_{\mathbb{R}^{n}} u^{r+1} d x d t
$$

And then

$$
\int_{0}^{t}\left(\int_{\mathbb{R}^{n}} u^{q} d x\right)^{\frac{p-1}{q}} \int_{\mathbb{R}^{n}} u^{r+1} d x d t \leq \int_{\mathbb{R}^{n}} u(x, t) d t \leq \pi^{\frac{n}{2}}\left(\pi^{\frac{n(p-1)}{2 q}}(4 n)^{-1}\right)^{-\frac{1}{p+r-m}}
$$

as $u_{0}(x, t) \geq 0$. This implies that

$$
\int_{0}^{\infty}\left(\int_{\mathbb{R}^{n}} u^{q} d x\right)^{\frac{p-1}{q}} \int_{\mathbb{R}^{n}} u^{r+1} d x d t<+\infty .
$$


On the other hand, from [25] we know that there exists $\delta>0$ such that the solution of (1.1) satisfies

$$
u(x, \tau)>\delta\left(1+B|x|^{2}\right)^{-\frac{1}{1-m}}
$$

for $B=\frac{(1-m) \alpha \delta^{1-m}}{2 m n}$ and some $\tau>0$. Setting

$$
\underline{u}(x, t)=\delta(1+t)^{-\alpha}\left(1+B|x|^{2}(1+t)^{-\frac{2 \alpha}{n}}\right)^{-\frac{1}{1-m}}
$$

with $\alpha=\frac{n}{2-n(1-m)}$, it is simple to verify

$$
\underline{u}(x, t) \leq u(x, t+\tau) \quad \text { for } x \in \mathbb{R}^{n}, t>0 .
$$

And we have

$$
\begin{aligned}
& \int_{0}^{\infty}\left(\int_{\mathbb{R}^{n}} u^{q}(x, t) d x\right)^{\frac{p-1}{q}} \int_{\mathbb{R}^{n}} u^{r+1}(x, t) d x d t \\
& \geq \int_{0}^{\infty}\left(\int_{\mathbb{R}^{n}} u^{q}(x, t+\tau) d x\right)^{\frac{p-1}{q}} \int_{\mathbb{R}^{n}} u^{r+1}(x, t+\tau) d x d t \\
& \geq \int_{0}^{\infty}\left(\int_{\mathbb{R}^{n}} \underline{u^{q}}(x, t) d x\right)^{\frac{p-1}{q}} \int_{\mathbb{R}^{n}} \underline{u}^{r+1}(x, t) d x d t \\
& =B^{-\frac{p+q-1}{2 q}} \delta^{p+r}\left(\int_{\mathbb{R}^{n}}\left(1+|\xi|^{2}\right)^{-\frac{q}{1-m}} d \xi\right)^{\frac{p-1}{q}} \int_{\mathbb{R}^{n}}\left(1+|\xi|^{2}\right)^{-\frac{r+1}{1-m}} d \xi \int_{0}^{\infty}(1+t)^{-1} d t \\
& =+\infty,
\end{aligned}
$$

since $-\alpha(p+r-1)+\frac{\alpha(p-1)}{q}=-1$ for $p=p_{c}$ and $\xi=\sqrt{B} x(1+t)^{-\frac{\alpha}{n}}$. This contradicts (2.8), and so our assumption that the solution of (1.1) globally exist for $t>0$ is not true, which proves Theorem 1.1 with $p=p_{c}$.

\section{Coexistence of global and non-global solutions}

This section mainly deals with the global solution for the case of $p>p_{c}$ to derive Theorem 1.2

Proof of Theorem 1.2 Firstly, we show that the solution of (1.1) must blow up in finite time for large initial data $u_{0}(x)$. The proof of Theorem 1.1 means that $u(x, t)$ does not exist globally, provided $u_{0}$ satisfies

$$
\left(\frac{k}{\pi}\right)^{\frac{n}{2}} \int_{\mathbb{R}^{n}} \mathrm{e}^{-k|x|^{2}} u_{0}(x) d x>\left(\pi^{\frac{n(p-1)}{2 q}}(4 n)^{-1}\right)^{-\frac{1}{p+r-m}} k^{\frac{2 q+n(p+1)}{2 q(p+r-m)}} .
$$

For any fixed $k=k_{0}>0$, we can choose large $u_{0}(x)$ to fulfil condition (3.1).

Next, we prove that the solution of (1.1) exists globally for any small initial data $u_{0}(x)$. Let

$$
\bar{u}=(t+1)^{-\beta}\left(D_{1}+D_{2}|x|^{2}(t+1)^{-\beta(1-m)-1}\right)^{-\frac{1}{1-m}},
$$


where $\beta=\frac{n(p-1)+2 q}{2 q(p+r-1)-n(1-m)(p-1)}$, and $D_{1}, D_{2}>0$ are to be determined. We demonstrate that $\bar{u}$ is a global supersolution of (1.1) for suitable $D_{1}$ and $D_{2}$. Setting

$$
Z=D_{1}+D_{2}|x|^{2}(t+1)^{-\beta(1-m)-1}=: D_{1}+D_{2} z
$$

with $z=|x|^{2}(t+1)^{-\beta(1-m)-1}$, we have

$$
\begin{aligned}
\bar{u}_{t}- & \Delta \bar{u}^{m}-\left(\int_{\mathbb{R}^{n}} \bar{u}^{q} d x\right)^{\frac{p-1}{q}} \bar{u}^{r+1} \\
= & (t+1)^{-\beta-1} Z^{-\frac{1}{1-m}-1}\left[-\beta Z+\frac{D_{2}(\beta-\beta m+1)}{1-m} z+\frac{2 m D_{2} n}{1-m} Z-\frac{4 m D_{2}^{2}}{(1-m)^{2}} z\right. \\
& \left.-(t+1)^{-\beta r+1}\left(\int_{\mathbb{R}^{n}}(t+1)^{-\beta q}\left(D_{1}+D_{2}|y|^{2}(t+1)^{-1-\beta(1-m)}\right)^{-\frac{q}{1-m}} d y\right)^{\frac{p-1}{q}} Z^{-\frac{r}{1-m}+1}\right] \\
= & (t+1)^{-\beta-1} Z^{-\frac{1}{1-m}-1} G(Z) .
\end{aligned}
$$

For $\max \left\{0,1-\frac{2}{n}+r\right\}<m<1, q \geq 1, r \geq 0$ implying $\frac{2 q}{1-m} \geq \frac{2}{1-m}>n$, there exists a constant $C>0$ such that

$$
\begin{aligned}
& \int_{\mathbb{R}^{n}}(t+1)^{-\beta q}\left(D_{1}+D_{2}|y|^{2}(t+1)^{-1-\beta(1-m)}\right)^{-\frac{q}{1-m}} d y \\
& =\int_{\mathbb{R}^{n}}(t+1)^{-\beta q+\frac{n+n \beta(1-m)}{2}}\left(D_{1}+D_{2}|w|^{2}\right)^{-\frac{q}{1-m}} d w \\
& \leq C(t+1)^{-\beta q+\frac{n+n \beta(1-m)}{2}} .
\end{aligned}
$$

Substituting the above inequity into the expression of $G(Z)$ in (3.2), and using $D_{2} z=Z-D_{1}$, $\beta=\frac{n(p-1)+2 q}{2 q(p+r-1)-n(1-m)(p-1)}$, we have

$$
\begin{aligned}
G(Z) \geq & -\beta Z+\frac{D_{2}(\beta-\beta m+1)}{1-m} z+\frac{2 m D_{2} n}{1-m} Z-\frac{4 m D_{2}^{2}}{(1-m)^{2}} z \\
& -C(t+1)^{-\beta(p+r-1)+\frac{n+n \beta(1-m)}{2 q}(p-1)+1} Z^{-\frac{r}{1-m}+1} \\
= & \left(-\beta+\frac{\beta-\beta m+1}{1-m}+\frac{2 m D_{2} n}{1-m}-\frac{4 m D_{2}}{(1-m)^{2}}\right) Z \\
& -\left(\frac{\beta-\beta m+1}{1-m}-\frac{4 m D_{2}}{(1-m)^{2}}\right) D_{1}-C Z^{-\frac{r}{1-m}+1} \\
=: & F(Z) .
\end{aligned}
$$

To describe $F(Z) \geq 0$ for some $D_{1}$ and $D_{2}$, we have to show (i) $F\left(D_{1}\right) \geq 0$ and (ii) $F^{\prime}(Z) \geq 0$ for $Z \geq D_{1}$.

(i) $F\left(D_{1}\right)=\left(-\beta+\frac{2 m D n}{1-m}\right) D_{1}-C D_{1}^{-\frac{r}{1-m}+1} \geq 0$ is equivalent to

$$
\begin{aligned}
& D_{1}^{-\frac{r}{1-m}} \leq \frac{1}{C}\left(-\beta+\frac{2 m D_{2} n}{1-m}\right), \\
& D_{2}>\frac{\beta(1-m)}{2 m n} .
\end{aligned}
$$




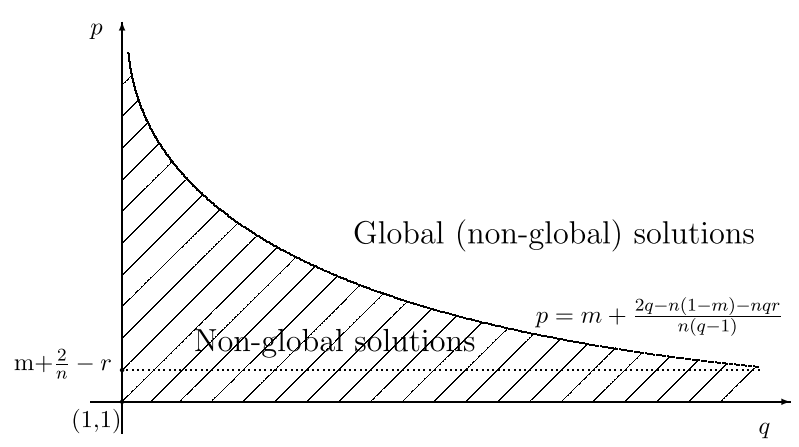

Figure 1 Critical Fujita exponent curve in $q-p$ plane

(ii) By simple computation, $F^{\prime}(Z)=-\beta+\frac{\beta-\beta m+1}{1-m}+\frac{2 m D_{2} n}{1-m}-\frac{4 m D_{2}}{(1-m)^{2}}-C\left(1-\frac{r}{1-m}\right) Z^{-\frac{r}{1-m}}$. If $1-\frac{r}{1-m} \leq 0$, condition (ii) is ensured by

$$
-\beta+\frac{\beta-\beta m+1}{1-m}+\frac{2 m D_{2} n}{1-m}-\frac{4 m D_{2}}{(1-m)^{2}}>0 .
$$

If $1-\frac{r}{1-m}>0$, condition (ii) is ensured by (3.6) and

$$
D_{1}^{-\frac{r}{1-m}} \leq \frac{1-m}{C(1-m-r)}\left(-\beta+\frac{\beta-\beta m+1}{1-m}+\frac{2 m D_{2} n}{1-m}-\frac{4 m D_{2}}{(1-m)^{2}}\right)
$$

Inequalities (3.5) and (3.6) require

$$
\frac{\beta(1-m)}{2 m n}<D_{2}<\frac{1-m}{2 m(2-n(1-m))}
$$

Due to $\beta=\frac{n(p-1)+2 q}{2 q(p+r-1)-n(1-m)(p-1)}$ and $p>p_{c}$, we can choose some $D_{2}>0$ that fulfils (3.8). For such $D_{2}$, choose $D_{1}>0$ large enough to satisfy (3.4) and (3.7).

In conclusion, $\bar{u}$ is a global supersolution to problem (1.1) with small initial data $u_{0}(x) \leq$ $\bar{u}(x, 0)=\left(D_{1}+D_{2}|x|^{2}\right)^{-\frac{1}{1-m}}$.

\section{Conclusion}

This paper shows that the model (1.1) possesses critical Fujita exponent $p_{c}=m+$ $\frac{2 q-n(1-m)-n q r}{n(q-1)}$ in Theorems 1.1 and 1.2, and we find that the coefficient $q$ of the nonlocal term affects the critical Fujita exponent. It's easy to see that $p_{c}$ is decreasing in $q$ with $\lim _{q \rightarrow \infty} p_{c}=m+\frac{2}{n}-r$ and $\lim _{q \rightarrow 1} p_{c}=\infty$. That is to say, the scope $1<p \leq p_{c}$ for the blow-up of any nontrivial solutions will be enlarged as $q$ is decreasing, and any nontrivial solution of (1.1) will blow up when $p>1$ and $q=1$. Refer to Fig. 1.

Acknowledgements

Not applicable.

\section{Funding}

This work was supported by the National Natural Science Foundation of China (Grant Nos. 11501438, 11501076), the Natural Science Basic Research Plan in Shaanxi Province of China (No. 2018JM1035), and the Young Talent fund of University Association for Science and Technology in Shaanxi, China (No. 20170607). 


\section{Competing interests}

The authors declare that they have no competing interests.

\section{Authors' contributions}

All authors contributed equally to this work. They all read and approved the final version of the manuscript.

\section{Author details}

${ }^{1}$ School of Science, Xi'an University of Architecture and Technology, Xi'an, P.R. China. ${ }^{2}$ School of Science, Dalian Ocean University, Dalian, P.R. China.

\section{Publisher's Note}

Springer Nature remains neutral with regard to jurisdictional claims in published maps and institutional affiliations.

\section{Received: 23 May 2019 Accepted: 14 October 2019 Published online: 21 October 2019}

\section{References}

1. Galaktionov, V.A., Levine, H.A.: A general approach to critical Fujita exponents in nonlinear parabolic problems. Nonlinear Anal. 34, 1005-1027 (1998)

2. Deng, W.B.: The blow-up rate for a degenerate parabolic equation with a non-local source. J. Math. Anal. Appl. 264(2), 577-597 (2001)

3. Anderson, J.R., Deng, K.: Global existence for degenerate parabolic equations with a non-local forcing. Math. Methods Appl. Sci. 20(13), 1069-1087 (1997)

4. Bebernes, J.W., Bressan, A.: Thermal behavior for a confined reactive gas. J. Differ. Equ. 44, 118-133 (1982)

5. Deng, K., Kwong, M.K., Levine, H.A.: The influence of non-local nonlinearities on the long time behavior of solutions of Burger's equation. Q. Appl. Math. 50, 173-200 (1992)

6. Deng, W.B., Li, Y.X., Xie, C.H.: Blow-up and global existence for a non-local degenerate parabolic system. J. Math. Anal. Appl. 277, 199-217 (2003)

7. Furter, J., Grinfeld, M.: Local vs. non-local interactions in population dynamics. J. Math. Biol. 27, 65-80 (1989)

8. Pao, C.V.: Blowing-up of solution for a nonlocal reaction-diffusion problem in combustion theory. J. Math. Anal. Appl. $166,591-600(1992)$

9. Souplet, P.: Blow-up in nonlocal reaction-diffusion equations. SIAM J. Math. Anal. 29, 1301-1334 (1998)

10. Du, L.L., Mu, C.L., Fan, M.S.: Global existence and non-existence for a quasilinear degenerate parabolic system with non-local source. Dyn. Syst. 20(4), 401-412 (2005)

11. Du, L.L.: Blow-up for a degenerate reaction-diffusion system with nonlinear nonlocal sources. J. Comput. Appl. Math. 202(2), 237-247 (2007)

12. García-Melián, J., Quirós, F.: Fujita exponents for evolution problems with nonlocal diffusion. J. Evol. Equ. 10(1), 147-161 (2010)

13. Bogoya, M., Ferreira, B., Rossi, J.D.: A nonlocal nonlinear diffusion equation with blowing up boundary conditions. J. Math. Anal. Appl. 337(2), 1284-1294 (2008)

14. Zhou, J.: The second critical exponent for a nonlocal porous medium equation in $\mathbb{R}^{N}$. Appl. Math. Lett. 38, 43-47 (2014)

15. Wang, S., Xie, C.H.: On critical exponent of blow-up for a nonlocal reaction-diffusion equations. Acta Math. Sin. 41, 261-266 (1998)

16. Fujita, H.: On the blowing up of solution of the Cauchy problem for $u_{t}=\Delta u+u^{\alpha+1}$. J. Fac. Sci., Univ. Tokyo 13, 109-124 (1966)

17. Aronson, D., Weinberger, H.F.: Multidimensional nonlinear diffusion arising in population genetics. Adv. Math. 30, 33-76 (1978)

18. Hayakawa, K.: On nonexistence of global solution os some semilinear parabolic equation. Proc. Jpn. Acad. 49, 503-505 (1973)

19. Kobayashi, K., Sirao, T., Tanaka, H.: On the blowing up problem for semilinear heat equations. J. Math. Soc. Jpn. 29, 407-424 (1977)

20. Deng, K., Levine, H.A.: The role of critical exponents in blow-up theorems: the sequel. J. Math. Anal. Appl. 243, 85-126 (2000)

21. Levine, H.A.: The role of critical exponents in blowup theorems. SIAM Rev. 32, 262-288 (1990)

22. Qi, Y.W.: On the equation $u_{t}=\Delta u^{\alpha}+u^{\beta}$. Proc. R. Soc. Edinb., Sect. A, Math. 123, 373-390 (1993)

23. Mochizuki, K., Mukai, K.: Existence and nonexistence of global solution to fast diffusions with source. Methods Appl. Anal. 2, 92-102 (1995)

24. Evans, L.C.: Partial Differential Equations. American Mathematical Society, Providence (1998)

25. Herrero, M., Pierre, M.: The Cauchy problem for $u_{t}=\Delta u^{m}$ when $0<m<1$. Trans. Am. Math. Soc. 291, 145-158 (1985) 